caveats on important clinical issues. He was also an effective manager of the service at Park Royal and in many ways this became an additional medium forensic secure unit in all but name.

Dr Gandhi was a man of razor-sharp intelligence who was very impatient to get things done. Although this was somewhat of a handicap when he was doing research, as he always seemed to know the answer before the research was completed, it was a tremendous asset in forensic mental health services, and the service could always rely on Naresh to find solutions when many others would have failed. He was always willing to give advice but very rarely needed to seek any himself.

Naresh continued to remain firmly and deliberately in the 'line of fire' throughout his working life. He worked excessively long hours and never shirked a new challenge, particularly if it involved a slight element of risk. This may have had a bearing on his final illness, as there was always some new enterprise pressing and as he was always optimistically convinced that everything was for the best, he failed to seek advice for his symptoms until he was very ill.

He married Alison, a Scot, in 1993 and had two sons, aged 10 and 16, of whom he was very proud. Naresh, as might have been predicted from his international spirit, readily embraced all aspects of his new culture with gusto and was equally at home with Indian and Scottish music, which had he lived, he would probably have tried to meld into a common form. He leaves a deep hole in forensic psychiatric practice and an even bigger one for his family, many friends and colleagues in North West London.

COID, J., KAHTAN, N., GAULT, S., et al (2001) Medium secure forensic psychiatry services. Comparison of seven English health regions. British Journal of Psychiatry, 178, 55-61.

PeterTyrer

\title{
reviews
}

\section{Oxford Handbook of Psychiatry}

David Semple, Roger Smyth, Jonathon Burns, et al Oxford: Oxford University Press, 2005, f24.95, pb, $953 \mathrm{pp}$. ISBN: 0-19-852783-7

The book has four sections: fundamentals of psychiatric practice; general adult psychiatry; psychiatric sub-specialties; and reference material. The clinical disorders are covered concisely under the headings of aetiology, epidemiology, clinical features, differential diagnoses, assessment/investigation, management and prognosis. All the psychiatric subspecialties have been adequately represented. The ICD-10 and DSM-IV coding index has also been provided.

The important information that a trainee in psychiatry needs on a day-today basis for the assessment and management of common and urgent situations, as well as medication with dosages and side-effects, has been adequately covered. The chapters on legal and ethical issues, mental health legislation in the UK and Republic of Ireland, and therapeutic issues deserve special mention as they provide precise information, which was previously difficult to find from a single source. The separate section on difficult and urgent situations will be an excellent aid for psychiatric trainees while on-call.

The book is bulky, which suggests that the fine balance between being overinclusive and being precise might not have been attained. However, this might change with later editions. The need for a chapter on evidence-based psychiatry in a handbook is a matter of debate. It is, however, a very well written chapter.
The title of the chapter 'Disorders of behaviour' suggests that in other clinical disorders there is no disorder of behaviour. The ICD-10 heading for this group of conditions 'Behavioural syndromes associated with physiological disturbance and physical factors' might have been more accurate. A few more blank pages for a reader to make their own notes would have been beneficial.

The authors need to be commended for this book which definitely measures up to the high standards set by other handbooks in the Oxford series. The simple note-based format makes for easy reading. It is a valuable resource for medical students during their psychiatry placements, senior house officers and, most importantly, those preparing for the MRCPsych examinations. Busy consultants and specialist registrars will find this book a useful quick reference and it will doubtless find a place in the bags of all psychiatric trainees.

Pavan Kumar Mallikarjun Queen Elizabeth Psychiatric Hospital, Mindelsohn Way, Edgbaston, Birmingham B15 2QZ, e-mail: pavan@doctors.net.uk

\section{OSCEs in Psychiatry}

\section{Ranga Rao}

London: Gaskell, 2005, £20.00

$208 \mathrm{pp}$.

ISBN: 1-904671-17-9

The objective structured clinical examination (OSCE) has revolutionised the way that we examine undergraduates and postgraduates in medicine. It is now widely used in medical schools and since spring 2003 has been an integral part of the MRCPsych part I examination. This book will be of major relevance to all trainees and should go a long way to demystifying the OSCE for their trainers.
The OSCE is introduced in some detail and its advantages (the provision of a uniform examination that deals with specific issues) and disadvantages (the scenarios may not mimic real-life situations) are acknowledged. The development of specific OSCEs is discussed and vital tips are then given on how to pass and train for the examinations.

A large part of the book is dedicated to specific examples of OSCEs. Several (but unfortunately not all) sub-specialties of psychiatry are covered as is neurological examination and even feedback of neurological investigations. Not surprisingly this part of the book is quite repetitive but covers a wide range of different scenarios and will doubtless be of value to those preparing for their part I examination and those responsible for designing OSCEs. Each example starts with constructive instructions to candidates, discusses the key points that should be covered and then describes what the authors would consider to be a good approach. The scenarios are fair and cover issues that one would expect in the exam. Any candidate who approached their OSCE in the way suggested by the authors should enhance their chance of success.

The book also contains an interactive CD-ROM. I suspect this will be of most value to trainees revising for exams and would certainly provide some variety, which is usually helpful.

This book is a worthy addition to the College Seminars Series, covering a key topic that all psychiatrists need to be familiar with. The authors are to be congratulated on a book that is comprehensive without being overinclusive.

Jonathan I. Bisson Clinical Senior Lecturer in Psychiatry, Monmouth House, University of Wales College of Medicine, Heath Park, Cardiff CF14 4XW 